\title{
The Influence of Friendship Network on Graduate Student's Research Potential
}

\author{
Xiao Liu ${ }^{1}$; Haozheng $\mathrm{Zhu}^{2}$ \\ ${ }^{1}$ Management school, Jinan University \\ ${ }^{2}$ College of economics, Jinan University
}

\begin{abstract}
Research activity is a major part of academic degree education for postgraduate student in China. A long line of research has examined the influence of social networks on individuals' behavior. This paper presents evidence on students' independent research behavior in searching for new knowledge, as embodied in the friendship tie among first-year graduate students. We describe the structure of the friendship network as a whole and present evidence on the determinants of social ties. Finally, we examine evidence on the extent to which individual graduate student becomes a broker integrated into a friendship network significantly influence a student's research potential.
\end{abstract}

Keywords: friendship network; research potential, social network analysis; ERGM; postgraduate education

\section{Introduction}

Postgraduate education involves learning and studying for degrees, professional or academic certificates, and it is normally considered to be part of higher education. There are two main types of qualifications studied for at the postgraduate level in China: vocational and academic degrees. Different with the former focusing on the course studying in a certain scientific field, research activity is a major part of academic degree education. Graduate student's education embraces both independent selfdirected study and collective share learning. Meanwhile, students supervised by their advisors who commonly related to research projects, involve more scientific research and experiment.

Educators suppose student's readiness to solve research problems of different kind. Research potential as an integrative characteristic of a person's abilities ensures achievement of personally important and socially significant aims in the field of scientific research in the past, present and future. ${ }^{[1]}$ There are three main components of a person's research potential, motivational, cognitive and behavioral component. ${ }^{[2]}$ Motivational component reveals itself in interest to research, in enjoying getting new knowledge. Cognitive component reveals itself in feeling of a lack of understanding of something, being sensitive to contradictions and productiveness of thinking, in the skill of putting clear questions and formulating persuasive suggestions. Behavioral component reveals itself in desire to meet the demand in knowledge by action, in the persistence and stamina in searching for new knowledge and in acquisition of new skills during research practice.

However, it is difficult to judge a student's research potential in a real world. Some educators indicate "doing well in classes does not mean that someone has the skills necessary to do research". And "doing research as a student typically means he/she have to be willing to interact with at least one other person. Some people can do this well and some people can't, no matter how high their GPA". ${ }^{33]}$

Recently decades, there is a growing body of studies emphasizing that individuals are embedded in their societies. The relationship shape a person's behavior or attitudes beyond the influence of his or her own individual characteristics ${ }^{[4]}$. Thus, the related social structure, though sometimes invisible, is often associated with instrumental outcomes, including power, innovation, learning outcomes, and job performance. ${ }^{[5]}$

Naturally, as this interest has grown, educational researchers have drawn on its interdisciplinary foundations to study a range of phenomena. ${ }^{[6]}$ Some researchers focused on the "relations" among the university students, such as friendship, advice, adversarial and communication, effect to an individual's performance. ${ }^{[7]}$ These studied provided evidences that the friendship and advice relations were positively related to a student's academic performance, and an adversarial network were negatively related to performance ${ }^{[7][8]}$. Furthermore, some researchers empirically demonstrated that appropriate arrangement of groups in a university course might increase the student's learning satisfaction and academic performance, according to each student's position in a social network. ${ }^{[8]}$

This study interests in postgraduate student's behavior in the first year after entering to a new university program, a critical turning point in high education for academic degree. In addition to considering a number of relevant attributes on student's (e.g., academic history, gender, etc.), to fully understand and model the student's behavior through student attending research activities (e.g., search for new knowledge, comment or review on other's research works), the relational data (i.e., student-to-student relations) 
inherent to the social network perspective should be investigated.

From a practical standpoint, the purpose of study is not only to discuss the topological structure of social network among students, but also to understand of the factors which effect student's position form in social network, the relationship between student's position and student's behavior, and then to design appropriate teaching strategies for improving the student's learning satisfaction and developing research potential.

The paper is organized into six sections. Section 2 describes our context and data. Section 3 describes the social ties of students and the network of ties as a whole. Section 4 presents evidence on the formation of friendships. Section 5 shows evidence on the effects of individual-level network position on student's independent research behavior and academic achievement. Section 6 concludes with limitations of our study and further research.

\section{Data source and measure method}

Data are drawn from 34 first year graduate students who are enrolled in the full-time program for academic certificates of business management at Jinan University, China. All students take a required the three-credit course on Management Theory during the fall semester of 2014, which is a combinational of traditional lecture, text-book discussions, academic paper reading and reviewing.

Due to all of them are in their first semester, and come from different university or different geography location, most of them are unacquainted with one another before entering this program, so their social networks develop gradually through various activities, including being roommates, attending lectures, doing group projects in class and after class. The "in-class" questionnaire is designed to collect or measure the social network data. With the beginning of the new school year, for grasping whether students know each other, the acquirement relations are measured by asking respondents the question "whom do you know?" At the end of term, the friendship relations are measured by asking respondents the question "who are the three best friends?" In addition, the survey also collect substantial information on student's socio-demographic attributes and behaviors.

The behavior component of research potential is measured according to the performance of student in acquiring new knowledge, such as literature searching and literature reviewing. As a first step to learn how do research, it is rather easier to review other's works than one's own work. Reviewing is pivotal in scientific activity, because the rank of a scientist depends not only on the level of his/her achievements, but also on an ability to understand the achievements of others. ${ }^{[9]}$ However, a post-graduate generally faces the reviewing problem because reviewing is not taught in China universities. Therefore, to their supervisors, frequently call in young post-graduates to review scientific articles, this may be of help at the time of defense of their dissertation.

All students are required to find two relevant academic articles published on the international journal according to their interest, read and understand, and then write an essay to review the authors' research problem and contribution, finally make a presentation in the class to share knowledge which he/she learn from reading articles. Literature searching performance is measured in term of the authority (i.e., published on the top journal or high citation) and novelty (i.e., published latest) of academic articles. Literature reviewing performance is assessed based on the reviewing quality and value.

Student's final-performance includes four components: literature searching performance, reviewing performance, homework and classroom performance. Classroom performance is measured by classroom presentation and participation in discussions. Five-point Likert-type scales are used for each of the items.

\section{Mapping social networks}

Graphs can reveal several interesting network properties, including whether relations are dense or spares, centralized or decentralized, or whether any individual members occupy strategically advantageous positions. So the first thing interested is to figure out who is at the center.

Figure 1 shows of complete networks of asymmetry friendship. Each node represents a student (diamond represents female, and triangle represents male). The lines are directed representing the direction of the friendship nomination. The double arrows edge represents a mutual friendship. The size of node represents the sum of in-degree and out-degree.

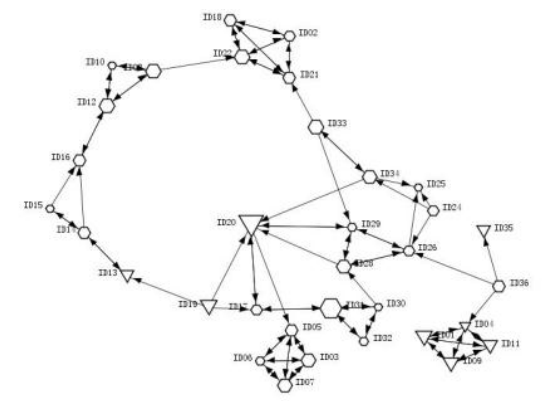

Fig. 1: complete networks of asymmetry friendship

The simplest and easiest way of measuring node centrality is accordingly by the degree of the different nodes in the network. A node in a position with high degree centrality can influence the group through knowing more people, and can be identified as the informal leaders of the group. Student 20 appears to receive more friendship nomination than others according the value of in-degree. 28 students of all students name three the best friends (i.e., outdegree), four students nominate two best friends, and two students nominate just only one best friends. 
It may also be interesting to find out who is on the periphery. Three students $(19,24$ and 36) are not nominated by his/her classmates, although they name three best friends. Both student 25 and 35 report having no friends, although they are named as a friend by other students. This suggests that such students are indeed more likely to be socially isolated within the class. But these peripheral members may have ties to other people outside class boundary. In these instances, the actor perhaps serves as bridge to other groups or network.

If we consider the network of mutual friendships, those dyads in which students nominate each other are likely to be stronger than one-way friendship nomination. There are $55 \%$ reciprocal friendships, and are slight gender tendency. Three cliques show same-sex friendship.

\section{Statistical inferences on form of network relations}

We now turn to understand the factors driving the formation of friendships in the class. For providing evidence on the mechanisms that lead to the formation of these within-school friendship, exponential random graph models (ERGMs) is used to identify the processes of network structure emergence and tie formation. ${ }^{[4]}$

ERGMs work by measuring a limited set of known statistics from a network and then use the distribution such statistics to generate random networks. These random networks are then compared to the observed networks to assess the likelihood of fit. ${ }^{[10]}$

We start the formal analyses with a baseline model including just the number of edges. Table 1 lists the interesting structure parameters, which are micro-level mechanisms that produce a higher-level network structure. Table 1. Structure Parameters of ERGMs

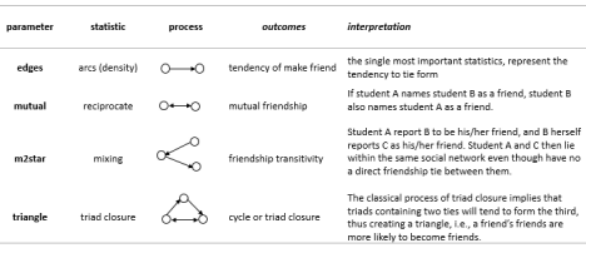

In addition to providing hypothesis testing on structural parameters (e.g., reciprocity or transitivity). ERGMs also permit actor's attributes to be incorporated into model estimation that including actor-level covariates such as those related to individuals' demographic (e.g., gender) or behavioral (e.g., attending team work) characteristics.

In our model, nodes (i.e., "actors") can be characterized by individual's attributes including gender, roommate, team work and advisor. "Nodematch" that can capture the network's tie form tendency based on the combination of individual's number of attributes. This is a dyad-level process by which common form is the greater propensity to partner with others having attribute similar to one's own.

Table 2 presents a number of analysis results. The model-3 shows that the structural features of mutual and triad closure are statistical significant positive effect on the probability of a tie form, transitivity is a significant negative effect. In addition, a significant positive effect on the probability of a tie based on the same attributes of roommate and advisor. That means, if two students have the same advisor, the more opportunity to study or discuss together, and the greater propensity to make friends. Meanwhile, if two students are roommates, the more possibility to attend the same activities, the greater propensity to friendship form. However, not only in teamwork matching, but also in sexual matching, the effect like common friendship pattern among adolescent students ${ }^{[4]}$, doesn't exists.

\begin{tabular}{|c|c|c|c|c|}
\hline Parameters & Model-1 & Model-2 & Model-3 & $\begin{array}{l}\text { Model-4 } \\
\text { M }\end{array}$ \\
\hline Edges & $-2.46(0.11)^{* \cdots}$ & $-3.49(30.22)$ & $-1.40(0.58)^{\circ}$ & $-1.08(0.52)^{*}$ \\
\hline mutual & & $3.81(\mathrm{NA})$ & $3.23(0.50)^{* * *}$ & $3.24(0.50)^{* * *}$ \\
\hline m2star & & $-0.26(1.53)$ & $-0.80(0.14)^{\cdots *}$ & $-0.81(0.13)^{\cdots} \cdots$ \\
\hline Triangle & & $0.66(\mathrm{NA})$ & $0.52(0.06)^{* * *}$ & $0.51(0.06)^{* * *}$ \\
\hline nodematch.team & & & $0.13(0.26)$ & \\
\hline nodematch.advisor & & & $1.64(0.43)^{\cdots} \cdots$ & $1.73(0.42) \cdots$ \\
\hline nodematch.gender & & & $0.41(0.31)$ & \\
\hline nodematch.roomate & & & $1.72(0.26) \cdots$ & $1.62(0.23)^{\cdots *}$ \\
\hline AIC & 1557 & 833.2 & 263.2 & 262.8 \\
\hline BIC & 1562 & 853.3 & 303.4 & 293 \\
\hline
\end{tabular}

Note: the value represent estimate and standard error. ' $* * *$ ' $\mathrm{p}<0.001$ '**' $\mathrm{p}<0.01$ '*' $\mathrm{p}<0.05$

We proceed to remove the non-significant terms to see if that can improve model fit. Accordingly, we fit our final model (see Model-4). The implication of the coefficients represents a conditional log-odds of two actors forming a tie. For example, the log-odds that a mutual tie (not add any structure pattern to the network) will form in the network equals 3.24, equaling a probability exp (3.24)/ (1+exp (3.24)), or 0.96 .

\section{Network factors on predicting research potential}

Friendship, which often begins with attraction to similar others, develops over time through shared experiences, frequent interaction, and growing affection. ${ }^{[11]}$ Because friendship is based on the emotional attachment and mutual positive affect ${ }^{[12]}$, enables friendship to support the access to information and knowledge directly and indirectly. A student who is central in a friendship network has a greater chance of helping others and being helped; thus, he/she is likely to perform better.

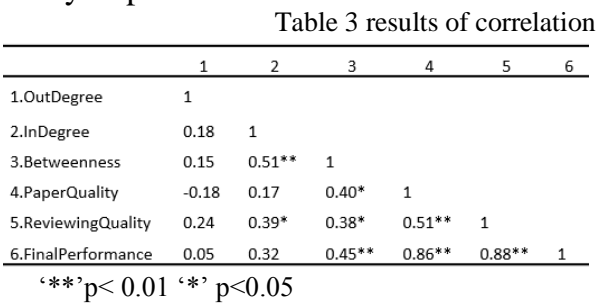

Person correlations are calculated among individual-level network centrality variable, student's research potential and academic achievement (Table 3).

Degree and closeness centrality are based on the reachability of a person within a network. The betweenenss centrality is defined as the portion of the number of shortest 
paths (between all pairs of nodes) that pass through the given node divided by the number of shortest path between any pair of nodes (regardless of passing through the given node).

Comparing with node's degree centrality, individual's betweenness centrality is more statistical significant positively to research potential and academic achievement.

Table 4 results of regression analysis

\begin{tabular}{lcccc}
\hline & $\begin{array}{c}\text { Partial } \\
\text { Coefficient }\end{array}$ & $\begin{array}{c}\text { Standard } \\
\text { Error. }\end{array}$ & $\mathrm{t}$ & $\begin{array}{c}\text { Significant } \\
\text { level }\end{array}$ \\
\hline Constant & 6.98 & 0.60 & 11.70 & $6.6 \mathrm{E}-13$ \\
InDegree & 0.17 & 0.24 & 0.70 & 0.487 \\
Betweenness & 0.03 & 0.02 & 2.08 & $0.046^{* *}$ \\
Overall adjusted R ${ }^{2}$ & 0.21 & & & \\
Overall $\mathrm{F}$ & 4.23 & & & 0.023 \\
\hline \multicolumn{1}{c}{ '**'p<0.01 '*' $\mathrm{p}<0.05$} & & &
\end{tabular}

Table 4 presents the results of regression analyses with an individual's final performance as the dependent variable and network structure variables as the independent variables. As shown in Table 3, betweenness was the best determinant of a graduate student's academic performance. Move to no significant in-degree variable, the betweeness factors could explain 14 percent of variance in paperquality and 11 percent in reviewing-quality, and 20 percent in final-performance.

Betweenness centrality of a node is often considered as an indicator of the power and influence these actors have in a group or organization. ${ }^{[12]}$ Individual with high betweeness centrality play the role of a broker or gatekeeper to connect the nodes and sub-groups.

Because research activity of individual dependents on the capability of communication with other persons, the more a person is a go-between, the more central his/her position in the network, the more his/her performance in searching or learning for new knowledge.

\section{CONCLUSIONS}

Application of social network analysis, as a powerful alternative methodology, to educational systems is a promising research area. The purpose of the present study is not only interested in describing student-to-student social tie properties but also providing some evidences on its formation and consequences.

However, there are several limitations to our study that should be addressed in future research. First, our relatively small sample size, limits the statistical power to detect the form mechanism of friendship networks. Second, with research activity increases, a student's social network environment influence his/her academic progress over time. Therefore, we should move beyond static snapshots to explore how attitudes and behaviors spread through networks over time, and how a friendship network changes over time and how these changes relate to outcomes. These interesting problems need to deeply investigate by understanding concepts such as centrality, bridging, group, position, and homophily.
The aware of the feedback of network effect is important to an educator, and helpful to design effective strategies to improve the research performance of postgraduate students by shaping their social tie. Such designs are very challenging but valuable in the educational practices.

\section{References}

[1] N.V.Bordovskaia, "Research potential and its manifestation among university students," Journal of International Scientific Publications: Educational Alternatives 10(2),pp.16-25,2012

[2] B.Nina and K.Svetlana, "Personal Features and research potential of students," The European Journal of Social \& Behavioral sciences, pp. 1284-1293, 2013.

[3] https://arjendu.wordpress.com/2009/01/14/you-cant-judge-researchpotential-by-classroom-performance/

[4] S.M.Goodreau, J.M.Kitts and M.Morris, "Birds of a feather, or friend of a friend? Using exponential random graph models to investigate adolescent social network," Demography 46(1), pp.103-125,2009

[5] D.Lusher and Masculinity, "educational achievement and social status: a social network analysis," Gender and Education 23(6), pp. 655-675, 2011

[6] C.E.Guldner and P.Stone-Winestock, "The use of sociometry in teaching at the university level," Journal of Group Psychotherapy, Psychodrama \& Sociometry 47(4),pp.177-186,1995

[7] Y.Heng-Li, T.Jih-Hsin,"Effects of social networks on student's performance: a web-based forum study in Taiwan," JALN 7(3),pp. 93-107,2003

[8] T.T.Baldwin, M.D.Bedell and J.L.Johnson, "The social fabric of a team-based M.B.A. program: network effects on student satisfaction and performance," Academy of Management Journal 40(6),pp.13691397,1997

[9] A.L.Fradkov, "How to publish a good article and to reject a bad one. Notes of a Reviewer," Automation and Remote Control,64(10),pp. 1643-1650, 2003

[10] S.M.Goodreau,"Advances in exponential random graph (p*) models applied to a large social network," Social Networks 29(2), pp.231-248, 2007

[11] D.E.Gibbons, "Friendship and advice networks in the context of changing professional values," Administrative Science Quarterly 49(2), pp. 238-262, 2004.

[12] R.T.Sparrowe, R.C.Liden and M.L.Kraimer, "Social network and the performance of individuals and groups," Academy of Management Journal 44(2),pp.316-325,2001 